Pacific Journal of Mathematic 


\title{
REPRESENTATION THEOREMS FOR CERTAIN FUNCTIONAL OPERATORS
}

\author{
R. E. EDWARDS
}

1. Introduction. Almost all the operators arising in applications of the Heaviside operational calculus share two properties. The precise formulation of these properties may vary, but their general nature is, in the first case, a commutativity rule relating to the operation of semitranslation, whilst in the second case it is a condition of continuity of some sort. Possible precise formulations of these conditions are typified by postulates $\left(O_{1}\right),\left(O_{2}\right)$ and $\left(O_{2}^{\prime}\right)$, which appear subsequently. Verification of the opening remark is to be found by glancing at the diverse illustrations of the technique to be found for example throughout [4].

It is the aim of the present paper to base proofs of general representation theorems upon such characteristic properties. The appropriate theorems will depend of course on the topologies envisaged in the continuity condition. Because of this, neither theorem proved here applies to all conceivable "operational expressions" : an outlaw expression would be $\exp (h p)(h>0)$, for instance. Modifications are possible, however, and would lead to theorems covering wider ranges of operational expressions.

As is well known, if the operands are restricted suitably, the operational calculus can be formulated in terms of the one-sided Laplace transform. Special attention is given to this case, and the corresponding representation theorem can be looked upon as a solution of the problem of factor functions for the Laplace transformation. The methods employed were suggested by those used in [3] to study factor functions for the Fourier transformation.

The general nature of all results obtained is very close to one given by L. Schwartz $[5$, p. 18 , Théorème X].

2. Classes of functions and operators. The widest class of functions to be considered will be denoted by $\mathscr{F}$ and will consist of those functions $f=f(t)$ which are defined and locally integrable on the halfline $R_{+}=\{t: t>0\}$. Functions which are equal a.e. are identified. A fundamental operator mapping $\mathscr{F}$ into itself is "semi-translation by $s$ ", where $s \geqq 0$ : this is denoted by $U_{s}$ and is defined by

$$
U_{s} f(t)= \begin{cases}f(t-s) & \text { for } t>s, \\ 0 & \text { for } 0<t \leqq s .\end{cases}
$$

The first of the two characteristic properties to be postulated about 
operators $T$ is

$\left(O_{1}\right) T$ commutes with $U_{s}$ for each $s \geq 0$.

The second, which reads

$\left(\mathrm{O}_{2}\right) \mathrm{T}$ is continuous from $\mathscr{F}$ into $\mathscr{F}$,

is interpreted relative to the topology of convergence in mean over each bounded interval of $R_{+}$. This topology on $\mathscr{F}$ is defined by the family of seminorms

$$
p_{n}(f)=\int_{0}^{n}|f(t)| d t \quad(n=1,2, \cdots)
$$

and makes $\mathscr{F}$ into a Fréchet space.

The first of the representation theorems may now be stated.

THEOREM 1. Let $T$ be a linear operator mapping $\mathscr{F}$ into itself which satisfies $\left(O_{1}\right)$ and $\left(O_{2}\right)$. Then $T$ is given by truncated convolution with a certain Radon measure $\mu$ concentrated on the closed half-line $t \geqq 0$, that is,

$$
T f(t)=\mu * f(t)=\int_{0}^{t} f(t-s) d \mu(s)
$$

for $f$ in $\mathscr{F}$. Conversely, if $\mu$ is such a measure, (2.3) defines an operator $T$ satisfyiug $\left(O_{1}\right)$ and $\left(O_{2}\right)$.

The measure $\mu$ may fail to be absolutely continuous; for this reason some care is needed in defining the right members of (2.3). This is dealt with in the proof of Theorem 1 , to be given in $\$ 3$.

The second theorem pays special attention to the subspace $\mathscr{E}$ of $\mathscr{F}$ composed of functions $f$ for which

$$
q_{n}(f)=\int_{0}^{\infty} e^{-n t}|f(t)| d t<+\infty
$$

holds for some $n$ which may depend on $f . \mathscr{E}$ is practically the largest domain for the Laplace transformation

$$
\hat{f}(p)=\int_{0}^{\infty} e^{-p t} f(t) d t ;
$$

if $f$ satisfies (2.4), then $\hat{f(p)}$ is defined for $9 x p \geq n$. Many of the operational expressions $F(p)$ appearing in applications of the Heaviside method act on $\&$ according to the ritual: take the Laplace transform, multiply by $F(p)$, and then invert the Laplace transform. The operational expression $F(p)$ thus acts as a "factor function". Detailed con- 
sideration of such factor functions is deferred until $\S 5$.

In order to state the second representation theorem it is necessary to introduce a topology on $\mathscr{E}$. If $\mathscr{E}_{n}(n=1,2, \cdots)$ denotes the subspace of $\mathscr{E}$ defined by the inequality (2.4), then $\mathscr{E}_{n} \subset \mathscr{E}_{n+1}$ and $\mathscr{E}=\bigcup_{n=1}^{\infty} \mathscr{E}_{n}$. Furthermore $q_{n}$ is a norm on $\mathscr{E}_{n}$ relative to which the latter is a Banach space. Accordingly, on $\mathscr{E}$ one may introduce the inductive limit topology defined by the $\mathscr{E}_{n}$ and the $q_{n}$; see $[1, \mathrm{p} .61]$ : this is the finest locally convex topology on $\mathscr{E}$ which induces on each $\mathscr{E}_{n}$ a topology less fine than that defined by the norm $q_{n}$. We shall denote by $\left(O_{2}^{\prime}\right)$ the condition which results from $\left(O_{2}\right)$ by replacing therein the Fréchet space $\mathscr{F}$ by the space $\mathscr{E}$ equipped with the said inductive limit topology.

THEOREM 2. Let $T$ be a linear operator mapping into itself which satisfies $\left(O_{1}\right)$ and $\left(O_{2}^{\prime}\right)$. Then $T$ admits a representation (2.3), where now the measure $\mu$ satisfies a condition

$$
\int e^{-n t} d|\mu|(t)<+\infty
$$

for some $n$ (which may depend on $\mu$, that is, on $T$ ); and conversely.

It may be noted here and now that Theorem 2 applies in particular to any $T$ satisfying $\left(O_{1}\right)$ and $\left(O_{2}\right)$ which happens to map $\mathscr{E}$ into $\mathscr{E}$. This is so because any such $T$ has a restriction to $\mathscr{E}$ which is necessarily continuous for $\mathscr{E}$ 's topology, which assertion is most easily established by applying the generalised closed graph theorem [2, p. 36, Exercice 13]. Condition $\left(O_{2}\right)$ is easily seen to imply that the restriction of $T$ to $\mathscr{E}$ has a closed graph when considered as a map of $\mathscr{E}$ into itself $^{1}$.

3. Proof of Theorem 1. The first thing is to define $\mu * f$ for $f \in$ $\mathscr{F}$ and any measure $\mu$ concentrated on the half-line $t \geqq 0$. An analogous process works in connection with Theorem 2 for functions $f \in \mathscr{E}$ and measures $\mu$ satisfying (2.6) for some $n$.

In the present case we note that for fixed $f$ in $\mathscr{F}, U_{s} f$ is a continuous function with values in $\mathscr{F}$ and that $p_{n}\left(U_{s} f\right)=0$ for $s \geqq n$. It is therefore certain that the abstract integral

$$
\int U_{s} f \cdot d \mu(s)
$$

exists as an element of $\mathscr{F}$ : this element is $\mu * f$. To see how this

1 It is necessary merely to observe that, for each $n$, the topology of $\mathbb{F}$ induces on $\ell_{n}$ a topology less fine than that defined by $q_{n}$. So, by definition, the inductive limit topology is finer than that induced on $\mathscr{G}$ by $\mathscr{F}^{\prime}$ topology. This being so, it is trivial to verify that the restriction of $T$ to $\mathscr{E}$ has a closed graph. 
definition is related to the "pointwise" one, we note that the dual of $\mathscr{F}$ may be identified with the space of bounded, measurable functions $\varphi$ on $R_{+}$which vanish a.e. outside bounded intervals, the linear form associated with such a $\varphi$ being given by

$$
\langle f, \varphi\rangle=\int_{0}^{\infty} f(t) \varphi(t) d t .
$$

Now the definition of (3.1) is such that for all $\varphi$ one has

$$
\langle\mu * f, \varphi\rangle=\int\left\langle U_{s} f, \varphi\right\rangle d \mu(s),
$$

so that by $(3.2)$

$$
\int_{0}^{\infty} \mu * f(t) \cdot \varphi(t) d t=\int d \mu(s) \int_{s}^{\infty} f(t-s) \varphi(t) d t
$$

for all $\varphi$. If $f(t-s)$, qua function of $s$, is integrable for $\mu$ over bounded intervals, and if $\int_{0}^{t} f(t-s) d \mu(s)$ is locally integrable (Lebesgue), the integral on the right can be rewritten as

$$
\int_{0}^{\infty} \varphi(t) d t \int_{0}^{t} f(t-s) d \mu(s) .
$$

Comparison shows that, under these conditions, $\mu * f$ is the function defined a.e. as $\int_{0}^{t} f(t-s) d \mu(s)$. This latter definition covers in particular the truncated convolution of two functions in $\mathscr{F}$.

Consider then the operator $T$ defined by $T f=\mu * f$. By what has been said, $T$ maps $\mathscr{F}$ into itself. Linearity of $T$ is obvious. Since also $U_{s} U_{a}=U_{a} U_{s}$ for $a \geqq 0, s \geqq 0$, and $U_{a}$ is continuous on $\mathscr{F}$, the abstract definition gives at once

$$
T U_{a} f=\int U_{s} U_{a} f \cdot d \mu(s)=\int U_{a} U_{s} f \cdot d \mu(s)=U_{a} \int U_{s} f \cdot d \mu(s)=U_{a} T f ;
$$

thus $T$ satisfies $\left(O_{1}\right)$.

To prove the continuity of $T$ it is merely necessary to take stock of the fact that $p_{n}\left(U_{s} f\right)$ vanishes for $s \geqq n$ and is everywhere at most $p_{n}(f)$. As a consequence,

$$
p_{n}(T f) \leqq \int p_{n}\left(U_{s} f\right) d|\mu|(s) \leqq m \cdot p_{n}(f),
$$

where $m$ is the $|\mu|$-measure of the interval $0 \leqq s \leqq n$. Thus $\left(O_{2}\right)$ is satisfied. The converse part of Theorem 1 is thus established.

Suppose now that $T$ satisfies $\left(O_{1}\right)$ and $\left(O_{2}\right)$. If $f$ and $g$ belong to $\mathscr{F}$ we have 


$$
\begin{aligned}
T(f * g) & =T\left(\int_{0}^{\infty} U_{s} g \cdot f(s) d s\right)=\int_{0}^{\infty} T U_{s} g \cdot f(s) d s \\
& =\int_{0}^{\infty} U_{s} T g \cdot f(s) d s=f * T g .
\end{aligned}
$$

This is applied to a sequence $g=g_{\nu}(\nu=1,2, \cdots)$ forming an "approximate identity" for the truncated convolution. A simple example of such a sequence is furnished by the functions

$$
g_{\nu}(t)= \begin{cases}\nu & \text { for } 0<t<1 / \nu \\ 0 & \text { for } t>1 / \nu\end{cases}
$$

It is easily verified that $f * g_{\nu} \rightarrow f$ in $\mathscr{F}$, and that $p_{n}\left(g_{\nu}\right) \leqq 1$ for all $n$ and all $\nu$. Since $T\left(f * g_{\nu}\right)=f * T g_{\nu}$, if we let $\nu$ tend to infinity there follows

$$
T f=\lim _{\nu \rightarrow \infty}\left(f * h_{\nu}\right),
$$

where $h_{\nu}=T g_{\nu}$. Now the sequence $\left(g_{\nu}\right)$ is bounded in $\mathscr{F}$ and $T$ is continuous; so the sequence $\left(h_{\nu}\right)$ is likewise bounded in $\mathscr{F}$, that is,

$$
\operatorname{Sup}_{\nu} \int_{0}^{n}\left|h_{\nu}(t)\right| d t<+\infty
$$

for each $n$. By dropping terms if necessary, we may assume that the sequence $\left(h_{\nu}\right)$ converges weakly to a measure $\mu$ concentrated on the halfline $t \geqq 0$. Accordingly, if $f$ is continuous, $f * h_{\nu}(t)$ will converge pointwise to $\int_{0}^{t} f(t-s) d \mu(s)$ for each $t$. However, $f * h_{\nu} \rightarrow T f$ in $\mathscr{F}$, and it follows at once that the two limits must coincide. Thus $T f=\mu * f$ holds at any rate for $f$ continuous. Such functions are dense in $\mathscr{F}$, and both members of this equality are continuous on $\mathscr{F}$. So equality holds for all $f$. This completes the proof of Theorem 1 .

4. Proof of Theorem 2. The general plan of the proof is very similar to that of Theorem 1. As before, the existence of the abstract integrals is dealt with first. In this connection it is useful to note the inequality

$$
q_{n}(\mu * f) \leqq q_{n}(\mu) \cdot q_{n}(f),
$$

where $q_{n}(\mu)$ denotes the left member of (2.6), provided both factors on the right are finite. Thus if $\mu$ satisfies (2.6) for a certain $n$, and if $f$ belongs to $\mathscr{C}_{N}$ for some integer $N$, then (4.1) shows that $\mu * f$ belongs to $\mathscr{E}_{M}$, where $M=\max (n, N)$. It shows also that the operator $T$ defined by $T f=\mu * f$ has the property that its restriction to each subspace $\mathscr{E}_{n}$ is continuous relative to the norm $q_{n}$. Hence $[1, \mathrm{p} .62] T$ is continuous 
from $\mathscr{E}$ into itself. In this way the converse part of Theorem 2 is established.

The direct part also runs much as before. The sequence $\left(h_{\nu}\right)$ is constructed again and will this time be bounded in $\mathscr{E}$. The limiting measure $\mu$ exists, but it remains to show that $\mu$ satisfies (2.6) for some $n$. This will follow as soon as it is shown that the $h_{\nu}$ lie in some $\mathscr{E}_{n}$, where $n$ is fixed independent of $\nu$, and remain bounded in $\mathscr{E}_{n}$. This does not follow directly from the boundedness of $\left(h_{\nu}\right)$ in $\mathscr{E}$ by virtue of $[2$, p. 8 , Proposition 6] since $\mathscr{E}$ is not a strict inductive limit. Nevertheless the desired result can be proved as follows.

Lemma. Let $B$ be a bounded subset of $\mathscr{E}$. There exists an integer $n$ such that $B \subset \mathscr{E}_{n}$ and $B$ is bounded relative to the norm $q_{n}$.

Proof. The dual of $\mathscr{E}$ may be identified with the space $\mathscr{B}$ of measurable functions $\varphi$ on $R_{+}$which satisfy

$$
r_{n}(\varphi)=\underset{t>0}{\operatorname{ess} \sup }\left|e^{n t} \varphi(t)\right|<+\infty
$$

for all $n$, the linear form associated with such a $\varphi$ is given by (3.2). Since $B$ is bounded in $\mathscr{E}$, the quantity

$$
Q(\varphi)=\sup _{h \in B}\left|\int_{0}^{\infty} h(t) \varphi(t) d t\right|
$$

is finite for each $\varphi$ in $\mathscr{D}$. Now $\mathscr{S}$, equipped with the seminorms $r_{n}(n=$ $1,2, \cdots)$, is a Fréchet space. Further $Q$ is a seminorm on $\mathscr{S}$ which is plainly lower semicontinuous, this last since $Q$ is expressly defined as the upper envelope of continuous seminorms. It follows from this that $Q$ is in fact continuous on $\int^{3}$. This signifies precisely that there is an integer $n$ and a number $C$ such that

$$
Q(\varphi) \leqq C \cdot r_{n}(\varphi)
$$

for all $\varphi$ in $C$ is independent of $\varphi$. Thus

$$
\left|\int_{0}^{\infty} h(t) \varphi(t) d t\right| \leq C \cdot \underset{t>0}{\operatorname{ess} \sup }\left|e^{n t} \varphi(t)\right|
$$

holds for all $\varphi$ in $\mathscr{S}$ and all $h$ in $B$. From this it is an easy deduction that

$$
\int_{0}^{\infty} e^{-n t}|h(t)| d t \leqq C
$$

for all $h$ in $B$, which is the result stated.

This lemma permits the proof of Theorem 2 to be effected. 
5. Factor Operators on $\because$. By a factor operator we shall mean one which is defined via a factor function for the Laplace transformation. The factor function $F(p)$ is assumed to be defined on some half-plane $\Re p \geqq n$, where $n$ may depend on $F$, and to have the property that, for each $f$ in $\mathscr{E}$ the function $F(p) \cdot \hat{f}(p)$ concides on some right-hand halfplane with the transform $\hat{g}(p)$ of some $g$ in $\mathscr{E}$. This $g$, whose existence is postulated, is then unique. The corresponding factor operator $T$ is then defined by $T f=g$.

Such a factor operator $T$ plainly satisfies $\left(O_{1}\right)$, but continuity of $T$ is not at all obvious. The relation

$$
T f * g=f * T g,
$$

which plays a crucial rôle on the above proofs, has hitherto been deduced from $\left(O_{1}\right)$ by means of continuity. In the case of a factor operator, (5.1) is verifiable right from the start due to basic properties of the Laplace transformation. This fact permits us to deduce continuity of $T$ and thus renders possible an appeal to Theorem 2 .

As we shall now see, continuity of $T$ will follow if (5.1) is known to hold for all $f$ and for all $g$ of a quite restricted class, say $G$. For this purpose we use again the generalised closed graph theorem. According to this, in order to show that $T$ is continuous it will suffice to show that: if a directed family $\left(f_{i}\right)$ converges to 0 in such a way that $T f_{i}$ converges to a limit, say $f$, then $f$ is necessarily 0 . However, we have seen in $\S 4$ that convolution is continuous in each factor, so that $T f_{i}$ $\rightarrow g$ implies $T f_{i} * g \rightarrow f * g$ for each $g$ in $\mathscr{E}$. Assuming that $g$ belongs to $G$, (5.1) permits this to be written $f_{i} * T g \rightarrow f * g$. Since $f_{i} \rightarrow 0$, the left member tends to 0 . Hence $f * g=0$ for all $g$ in $G$. If this holds, even for quite small classes $G$, it follows that $f=0$.

In this way we see from Theorem 2 that the factor functions $F$ are precisely those which are themselves Laplace transforms of measures $\mu$ satisfying (2.6) for some $n$.

\section{REFERENCES}

1. N. Bourbaki, Eléments de Muthématique, Espaces Vectoriels Topologiques, Chap. I-II (Actual. Scient. et Ind., $\mathrm{n}^{\circ}$ 1189, Paris, Hermann, 1953).

2. , Eléments de Mathématique, Espaces Vectoriels Topologiques, chap. III-V (Actual. Scient. et Ind., $\mathrm{n}^{\circ}$ 1229, Paris, Hermann, 1955).

3. R. E. Edwards, On factor functions, Pacific J. Math. 5 (1955), 367-378.

4. Sir Harold Jeffreys and Lady Jeffreys, Methods of mathematical physics (3rd ed.' Cambridge, 1956). 



\title{
PACIFIC JOURNAL OF MATHEMATICS
}

\author{
EDITORS
}

H. L. Royden

Stanford University

Stanford, California

R. A. Beaumont

University of Washington

Seattle 5, Washington
A. L. Whiteman

University of Southern California

Los Angeles 7, California

E. G. Straus

Unıversity of California

Los Angeles 24, California

\section{ASSOCIATE EDITORS}

\author{
E. F. BECKENBACH \\ C. E. BURGESS \\ M. HALL \\ E. HEWITT
}

\author{
A. HORN \\ V. GANAPATHY IYER \\ R. D. JAMES \\ M. S. KNEBELMAN
}

L. NACHBIN
I. NIVEN
T. G. OSTROM
M. M. SCHIFFER

G. SZEKERES

F. WOLF

K. YOSIDA

\section{SUPPORTING INSTITUTIONS}

UNIVERSITY OF BRITISH COLUMBIA

CALIFORNIA INSTITUTE OF TECHNOLOGY

UNIVERSITY OF CALIFORNIA

MON'TANA STATE UNIVERSITY

UNIVERSITY OF NEVADA

OREGON STATE COLLEGE

UNIVERSITY OF OREGON

UNIVERSITY OF SOUTHERN CALIFORNIA

\author{
STANFORD UNIVERSITY \\ UNIVERSITY OF UTAH \\ WASHINGTON STATE COLLEGE \\ UNIVERSITY OF WASHINGTON \\ AMERICAN MATHEMATICAL SOCIETY \\ CALIFORNIA RESEARCH CORPORATION \\ HUGHES AIRCRAFT COMPANY \\ THE RAMO-WOOLDRIDGE CORPORATION
}

Mathematical papers intended for publication in the Pacific Journal of Mathematics should be typewritten (double spaced), and the author should keep a complete copy. Manuscripts may be sent to any of the editors. All other communications to the editors should be addressed to the managing editor, E. G. Straus at the University of California, Los Angeles 24, California.

50 reprints per author of each article are furnished free of charge; additional copies may be obtained at cost in multiples of 50 .

The Pacific Journal of Mathematics is published quarterly, in March, June, September, and December. The price per volume (4 numbers) is $\$ 12.00$; single issues, $\$ 3.50$. Back numbers are available. Special price to individual faculty members of supporting institutions and to individual members of the American Mathematical Society: $\$ 4.00$ per volume; single issues, $\$ 1.25$.

Subscriptions, orders for back numbers, and changes of address should be sent to Pacific Journal of Mathematics, 2120 Oxford Street, Berkeley 4, California.

Printed at Kokusai Bunken Insatsusha (International Academic Printing Co., Ltd.), No. 10, 1-chome, Fujimi-cho, Chiyoda-ku, Tokyo, Japan.

PUBLISHED BY PACIFIC JOURNAL OF MATHEMATICS, A NON-PROFIT CORPORATION

The Supporting Institutions listed above contribute to the cost of publication of this Journal, but they are not owners or publishers and have no responsibility for its content or policies. 


\section{Pacific Journal of Mathematics}

\section{Vol. 7, No. $3 \quad$ March, 1957}

Silvio Aurora, Multiplicative norms for metric rings............... 1279

Ross A. Beaumont and John Richard Byrne, On the construction of

$R$-modules and rings with polynomial multiplication ............ 1305

Fred Brafman, An ultraspherical generating function . . . . . . . . . . . 1319

Howard Ernest Campbell, On the Casimir operator ............... 1325

Robert E. Edwards, Representation theorems for certain functional

operators..................................... 1333

Tomlinson Fort, The five-point difference equation with periodic

coefficients ..................................... 1341

Isidore Heller, On linear systems with integral valued solutions......... 1351

Harry Hochstadt, Addition theorems for solutions of the wave equation in

parabolic coordinates ................................ 1365

James A. Hummel, The coefficient regions of starlike functions . . . . . . . . 1381

Fulton Koehler, Estimates for the eigenvalues of infinite matrices ......... 1391

Henry Paul Kramer, Perturbation of differential operators ............. 1405

R. Sherman Lehman, Development of the mapping function at an analytic

corner .......................................... 1437

Harold Willis Milnes, Convexity of Orlicz spaces.................. 1451

Vikramaditya Singh, Interior variations and some extremal problems for certain classes of univalent functions . . . . . . . . . . . . . . . . 1485

William Lee Stamey, On generalized euclidean and non-euclidean spaces ............................................. 1505

Alexander Doniphan Wallace, Retractions in semigroups .............. 1513

R. L. Wilder, Monotone mappings of manifolds .................. 1519 